\title{
Effect of Low Level Laser versus McKenzie Technique in Treatment of Chronic Low Back Pain
}

\author{
Tarek $\mathrm{F}^{1}$, Osama $\mathrm{FA}^{2 *}$, Mohamed $\mathrm{S}^{2}$ and Ragia $\mathbf{M K}^{3}$ \\ ${ }^{1} \mathrm{MOH}$, Shoubra Public Hospital, Hospitals and Medical Centers, Cairo University, Egypt \\ ${ }^{2}$ National Institute of Laser Enhanced Sciences, Cairo University, Egypt \\ ${ }^{3}$ Faculty of Physical Therapy, Cairo University, Egypt
}

*Corresponding author: Osama FA, National Institute of Laser Enhanced Sciences, Cairo University, Egypt, Tel: 20235676105 ; E-mail: o.fekry@hotmail.com

Received date: May 5, 2017; Accepted date: May 16, 2017; Published date: May 25, 2017

Copyright: @ 2017 Tarek F, et al. This is an open-access article distributed under the terms of the Creative Commons Attribution License, which permits unrestricted use, distribution and reproduction in any medium, provided the original author and source are credited.

\begin{abstract}
Background: Lower back pain refers to the pain and discomfort, which is usually, localized in between costal margin and inferior gluteal folds. Occasionally it is accompanied with pain in the leg too.
\end{abstract}

Patients: Forty five patients with chronic low back pain (mean age $40.5 \pm$ years, height $167 \pm \mathrm{cm}$, weight $82.5 \pm \mathrm{kg}$, and BM $29 \pm \mathrm{kg} / \mathrm{m}^{2}$ ) participated in this study.

Patients and methods: Patients were categorized into 3 different equal groups (15 nos); group (A) received low level laser, group $(B)$ received McKenzie technique and group $(C)$ received traditional electrotherapy and functioned as the control group. Data of visual analogue scale (VAS), patient specific functional Scale and (ROM) scores were collected from each patient pre and post the administration of physiotherapy.

Results: Statistical analysis using paired student t-test and unpaired student t-test showed significant differences in the VAS and PSFS values recorded pre and post treatment in both groups $A$ and B. There was significant difference in improvement in pain; ROM and functional disability in laser group (A) than in McKenzie technique group (B).

Conclusion: The physiotherapy program is not only effective for the patients having chronic lower back pain, it also helped in improving functional disability of the patients, especially when low level laser or McKenzie technique are added to the treatment program.

Keywords: McKenzie technique; Peripheral nociceptors; Proliferation; musculoskeletal

\section{Introduction}

Lower back pain is denoted by the pain and discomfort occurred in between the costal margin and inferior gluteal folds. Often, this pain is also accompanied by varied level of leg pain. Acute incidences of low back pain persist for less than 6 weeks. The sub-acute low back pain usually persists till 6 to 12 weeks; the chronic type of lower back pain is known to persist 12 weeks or more depending on the condition [1].

The Low Level Laser (LLL) treatment accelerates the overall healing process through interaction with the targeted tissues while using precise wavelengths of light. Implementation of this treatment regime can be done for those patients who are suffering from various degree of acute to chronic conditions. This treatment may aid in eliminating the pain, reducing the existing swelling and spasms and restoring the normal body functionality and movement [2].

\section{LLL may reduce pain by two mechanisms}

1- Anti-inflammatory -LLL reduces oxidative stress: Oxidative stress is generally caused through the stress causing to the mitochondria or tissues which remains in the ischemic conditions and in turn produces nitric oxide. In the cascading step of the process, this released nitric oxide binds with the cytochrome oxidase enzyme through a competitive inhibiting binding mode while replacing the oxygen molecule. As a consequence of this cellular event reduction in ATP production occurs. This whole scenario of the stress induction and modulation is also related to other conditions including specific wavelength of light etc. All these factors involved determine the functioning rate of cytochrome $c$ oxidase and helps in increasing the ATP production and diminishing the oxidative stress. A series of molecular events pertaining to the downstream metabolic effects allows the reduction of the interleukin $1 \beta$, prostaglandin E2, tumor necrosis factor $\alpha$ and other inflammatory markers [3].

Nerve blockage by 2- analgesia- LLL: Induction of analgesic effect can be augmented by comparatively higher irradiance energy based treatment which is physiologically happened through the disruption of the axonal transport in the small fibers like nociceptors. In this process there is a transient inhibition occurs in transmission of A-delta and C fiber. Afferent inputs from these peripheral nociceptors become diminished and allow modulation of the reorganization of the synaptic connections. Thus, central sensitization can be lowered through repetitive treatment regime [2]. 
Low-back pain (LBP) turned out to be one of the major health issues in urban societies. $58 \%$ to $70 \%$ of the population experiences LBP at some point in their lives [3]. Each year, $5 \%$ to $10 \%$ of the work force is off work because of their LBP, a majority of which usually lasts less than seven days. Almost $90 \%$ of all patients with acute LBP get better quite rapidly, regardless of therapy. The remaining $10 \%$ are at risk of developing chronic pain and disability. LBP induced back incapacity also accounts for more than $90 \%$ of social costs [4].

Robin McKenzie initiated a massive program which was named as McKenzie Program in 1960. He was a renowned physiotherapist from New Zealand. This particular program follows specialized treatment, assessment and prevention methodologies along with various exercises which aids in mobilization of the centralized pain and relieve the condition of the patient.

As part of the program important exercise such as side glides, extensions, various rotations, and flexions are used to ease the pain. Among these exercises, rotations and side glides are occasionally used for disc centralization before any extension work out followed by flexion category exercises [1].

In modern day pain diagnosis, especially that originates from the back and neck and spine, the McKenzie method is useful. The treatment regime considers patient involvement very actively for educating them in relevance to problems of back and neck. To diagnose the problem and strategize the treatment accurately, evaluation by a McKenzie therapist can become crucial. To ascertain the proper treatment, the therapist continuously repeats the process till satisfaction level is reached. Proper use of this treatment method helps in avoiding expensive diagnosis procedures such as imaging. Moreover, patients receive excellent assistance by the McKenzie therapists to understand the nature of the symptoms, exact required recommendation for proper exercises, maintaining perfect body positions etc. to address the root cause of the health issues and restore their normal movement and body function [5].

\section{Patients and Methods}

Forty five male and female patients with age ranging from 40 to 60 years were recruited from the Ain Shams General Hospital. All of them received treatment three days a week for four weeks. They were divided randomly and equally into two study groups and one control group as follows:

Group a: 15 patients who received traditional electrotherapy program (TENS, hot packs) in addition to low level laser treatment.

Group b: 15 patients who received traditional electrotherapy program based treatment along with the McKenzie treatment.

Group c: 15 patients who received traditional electrotherapy program served as a control group.

\section{Factors for inclusion and exclusion criteria of the patients:}

Patients having LBP pain for 6 months and more and having their age between 40 and 60 years, body weight in the range of 70 to $100 \mathrm{~kg}$, no previous history of spine surgery, no neurological defects and no rheumatoid arthritis.

The patients selected were based on their history and medical exams. At first, demographic data such as age, weight, sex, height, BMI, pain ROM and function specifications were assessed and documented. Pain assessment was based on Visual Analogue Scale (VAS) and ROM measured by OB Goniometer. Function was assessed by patient specific functional Scale (PSFS) for ADL, Schober test was used to examine deformities of lumbar spine. Low level laser (Ga-As) can penetrate and effect on the tissue up to the depth of $1-5 \mathrm{~cm}$. These specific studies include patients who were treated with laser. The frequency of the treatment was 3 times per week and it was continued for 4 weeks, mode of action was continuous infrared light with wavelength $904 \mathrm{~nm}$, low intensity 4J. Output power of $75 \mathrm{~mW}$, Power density $10 \mathrm{~mW} / \mathrm{cm}^{2}$, Exposure time $120 \mathrm{sec} /$ point, Spot area $1.1 \mathrm{~cm}^{2}$, treatment with contact application technique on lumbo-sacral region Para spinal muscle. Patients of McKenzie technique treatment group were treated with 5 exercise procedures.

Procedure 1- prone lying: The patients lied on their abdomen with their head turned to one side.

Procedure 2- prone lying in extension: The patients placed the elbow under the shoulders and raised the top half of their body, using elbow and forearm support while the hips and pelvis remained on the bed: The patient were asked to relax in this position, allowing the lower back to sag into more extension. The position was sustained for one minute or more.

Procedure 3- prone lying with extension elbow: The patients placed the elbow fully extended under the shoulders and raised the top half of their body. The position was sustained for one minute or more.

Procedure 4- extension in lying: The patients were allowed to rest in lying position and they were suggested to keep their hands and palms down. They were allowed to raise only the upper half of their body while straightening their arms. They were suggested to maintain the posture by relaxing their pelvis and thighs and were requested to sag with gravity. The upper body portion was then lowered to the plinth. This exercise was then repeated for about ten to fifteen times.

Procedure 5- extension in standing: The patients stood with their feet placed at shoulder width apart with a good base of support and felt stable when they performed the procedure. The hands were placed back across the belt line with the fingers pointing downwards. The patient then leaned backwards as far as possible, letting the head tip back, using the hands as a fulcrum by pressing them into the spine. After arching backwards the patients returned to a neutral standing, and the exercise was repeated about ten times. The patients of the McKenzie technique treatment group were treated thrice weekly and continued for a month long period.

Patients of the control group were treated through traditional physio-therapy modalities (TENS and hot pack) at weekly thrice for a month. Statistical analysis performed by using ANOVA and t-test with $\mathrm{P}<0.05$ significant.

\section{Result}

Statistical analysis was done using SPSS v. 23. Paired sample t-test was done to detect any significant difference between pre and posttreatment in each study group. When the three groups were compared based on age, height, weight and BMI, there were not much significant difference observed between the pre and post treated groups.

Group A: The obtained results of the descriptive study is expressed through mean $\pm S D$ for the pre and post treatment which revealed significant differences in VAS (Pain), F.Pt standing, F-Pt walking and F-Asc-Desc values $(p=0.0001)$. For ROM-E, ROM-F, ROM-Rt and 
Page 3 of 5

ROM-Lt, the observation displayed no significant variation in between the pre and post treatment as $\mathrm{p}=0.546,0.25$ and 0.879 respectively.

Group B: The descriptive data results expressed as mean \pm SD for pre and post treatment revealed very high significant differences for F-Pt standing $(\mathrm{p}=0.003)$, F-Pt walking $(\mathrm{p}=0.0001)$ and F-Asc-Desc $(\mathrm{p}=0.005)$. Regarding VAS (pain) and ROM-F, the results showed high significant difference in pre and post treatment $(\mathrm{p}=0.023$ and 0.011 ) respectively. For ROM-E, ROM-Rt and ROM-Lt the obtained results showed no important changes in pre and post treatment, $\mathrm{p}=0.4,0.887$ and 0.872 respectively.

Group C: The observation are expressed via mean \pm SD for pre and post treatment that showed considerable alterations for F-Pt standing, F-Pt walking and F-Asc-Desc $(\mathrm{p}=0.0001)$. However the obtained data showed high significant results for VAS (pain) $(\mathrm{p}=0.019)$ and ROM-Lt ( $\mathrm{p}=0.011$ ), but for ROM-Rt and ROM-F there were no significant results as $p=0.898$ and 0.559 respectively. ANOVA test was done to compare the obtained results for the three groups, followed by Post hoc tests that were run to confirm where the differences occurred between groups. The obtained results showed an overall significant difference in group i.e., a significant one-way ANOVA result. Post-hoc tests attempt to control the experiment wise error rate (usually alpha $=0.05$ ) in the same manner that the one-way ANOVA is used, instead of multiple ttests. For all obtained pre-treatment data, all outputs exhibited no noteworthy changes between groups $\mathrm{A}, \mathrm{B}$ and $\mathrm{C}$ as $\mathrm{p}>0.05$. For all obtained post-treatment data, all results had revealed substantial alterations between groups $\mathrm{a}, \mathrm{b}$ and $\mathrm{c}$ as $\mathrm{p} \leq 0.05$. Post hoc test was done as mentioned above to confirm where the differences occurred in groups mean the obtained results showed:

Pain: The results presented important alterations between gp (A) and gp (B) p=0.001 and between gp (A) and gp (C) p=0.002, that resulted in more significant difference between gp (A) and gp (B) more than between gp (A) and gp (C), however no significant differences between gp (B) and gp (C).

ROM-F: Comparing gp (A) with gp (B) and gp (C) the obtained results revealed the same significant differences as $p=0.029$, but the comparison between gp (B) and gp (C) showed no significant differences as $\mathrm{p}>0.05$ (Figures 1-3).

ROM-E, ROM-Rt and ROM-Lt: The obtained results showed no significant differences between the three groups as $\mathrm{p}>0.05$.

F-Pt standing: Comparing between gp (A) and gp (B) the obtained results showed that there were high significant differences as $p=0.017$. Also there were very high significant differences between gp (A) andgp (C) as $p=0.0001$. However the results showed no significant differences as $\mathrm{p}>0.05$.

F-Pt walking: Comparing between gp (A) and gp (B) the obtained results showed that there were high significant differences as $\mathrm{p}=0.002$ and very high significant differences between gp (A) and gp (C) as $\mathrm{p}=0.0001$. However the results showed no significant differences as $p>0.05$. It appeared that the differences effect was due to gp (A).

F-Asc-Desc: Comparing between gp (A) and gp (B) the obtained results showed that there were very high significant differences as $\mathrm{p}=0.0001$ and very high significant differences between gp (A) and gp (C) as $\mathrm{p}=0.0001$. However the results showed no significant differences as $\mathrm{p}>0.05$ (Table 1 ).

\begin{tabular}{|l|l|l|l|l|}
\hline Group & Assessment & Pre treatment & $\begin{array}{l}\text { post } \\
\text { treatment }\end{array}$ & P-Value \\
\hline
\end{tabular}

\begin{tabular}{|c|c|c|c|c|}
\hline & & Mean $\pm S D$ & Mean \pm SD & \\
\hline \multirow{8}{*}{ group a } & Pain & $6 \pm 1.6$ & $3.67 \pm 0.6$ & $0.0001^{*}$ \\
\hline & ROM-F & $41.07 \pm 5.2$ & $47.8 \pm 3.7$ & $0.0001^{*}$ \\
\hline & ROM-E & $13.67 \pm 1.8$ & $13.53 \pm 2$ & 0.85 \\
\hline & ROM-Rt & $20.2 \pm 2.24$ & $18.87 \pm 5.5$ & 0.389 \\
\hline & ROM-Lt & $20.73 \pm 1.5$ & $20.8 \pm 2.6$ & 0.932 \\
\hline & F-Pt Standing & $2.8 \pm 0.9$ & $6.27 \pm 0.9$ & $0.0001^{* \star *}$ \\
\hline & F-Pt Walking & $3.4 \pm 1.2$ & $7.07 \pm 0.8$ & $0.0001^{* *+}$ \\
\hline & F-Asc and Desc & $3.53 \pm 1.1$ & $6.8 \pm 1$ & $0.0001^{* *+1}$ \\
\hline \multirow{8}{*}{ group b } & Pain & $6.07 \pm 1.2$ & $5.47 \pm 1.3$ & 0.204 \\
\hline & ROM-F & $40.8 \pm 5$ & $42.73 \pm 5.6$ & 0.327 \\
\hline & ROM-E & $13.6 \pm 3.02$ & $13.27 \pm 2.5$ & 0.743 \\
\hline & ROM-Rt & $20.33 \pm 2.1$ & $20.4 \pm 2.85$ & 0.942 \\
\hline & ROM-Lt & $20.73 \pm 1.3$ & $20.67 \pm 2$ & 0.914 \\
\hline & F-Pt Standing & $3.87 \pm 1.96$ & $5.2 \pm 1.3$ & $0.035^{\star}$ \\
\hline & F-Pt Walking & $4.13 \pm 1.84$ & $5.93 \pm 1.1$ & $0.003^{* *}$ \\
\hline & F-Asc and Desc & $4.13 \pm 1.1$ & $5.2 \pm 0.8$ & $0.004^{* *}$ \\
\hline \multirow{8}{*}{ group c } & Pain & $5.93 \pm 1.5$ & $5.27 \pm 1.5$ & 0.237 \\
\hline & ROM-F & $40.6 \pm 5.8$ & $42.73 \pm 6$ & 0.331 \\
\hline & ROM-E & $13.6 \pm 2.5$ & $13.4 \pm 2.2$ & 0.818 \\
\hline & ROM-Rt & $20.33 \pm 1.45$ & $20.4 \pm 2.3$ & 0.924 \\
\hline & ROM-Lt & $20.67 \pm 1$ & $21.07 \pm 2.4$ & 0.559 \\
\hline & F-Pt Standing & $2.8 \pm 0.9$ & $4.47 \pm 0.83$ & $0.0001^{* * *}$ \\
\hline & F-Pt Walking & $3.47 \pm 0.83$ & $5.27 \pm 0.6$ & $0.0001^{* * *}$ \\
\hline & F-Asc and Desc & $3.47 \pm 1.1$ & $5.07 \pm 0.9$ & $0.0001^{* * *}$ \\
\hline
\end{tabular}

Table 1: Descriptive analysis for group A, B and C in both pre and post-treatment. 
Page 4 of 5

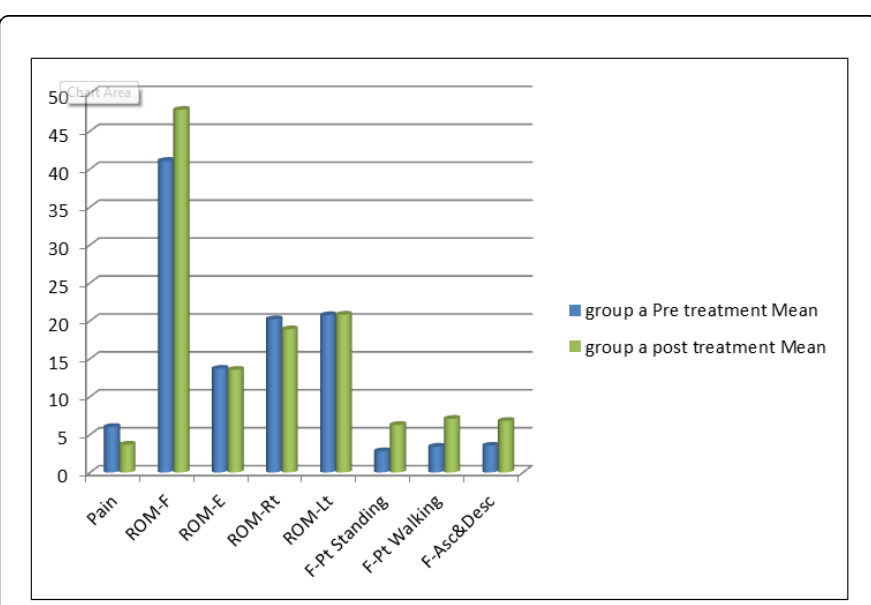

Figure 1: Comparison between pre and post assessment results of group A.

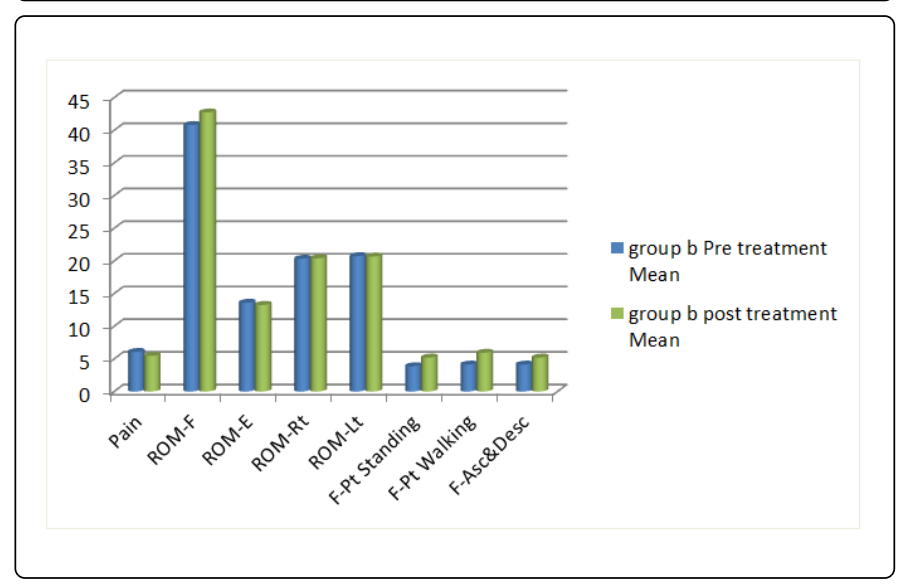

Figure 2: Comparison between pre and post assessment results in group B.

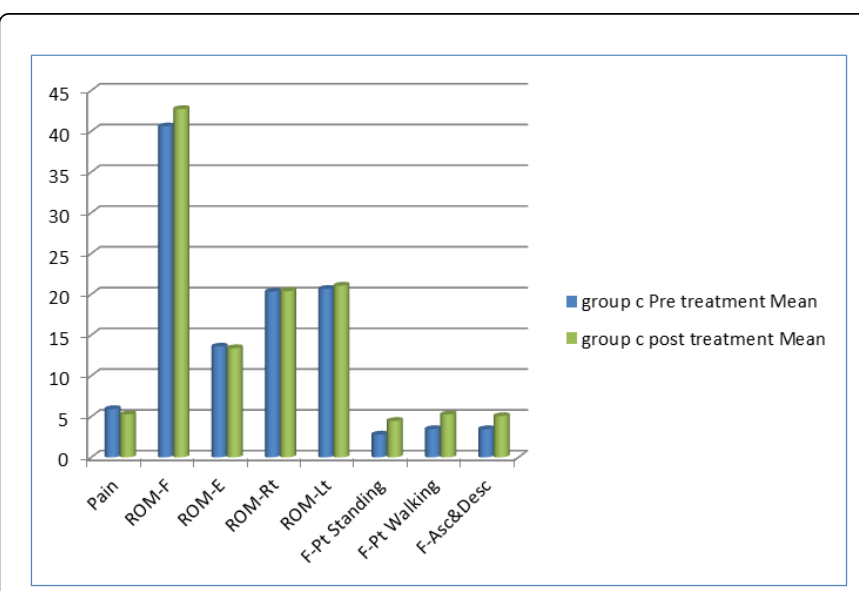

Figure 3: Comparison between pre and post assessment results in group $\mathrm{C}$.

\section{Discussion}

The current study aimed to evaluate the effect of Mackenzie exercise, and low level laser (LLL) treatment by employing clinical parameters for the patients having chronic low back pain. The patients were assessed for pain, ROM and functional assessment. This study was conducted at the out clinic of Ain Shams General Hospital.

Following treatment, the laser group (A) demonstrated highly significant improvement $(\mathrm{P}<0.001)$ in pain intensity that was measured by VAS (P-Value was 0.001 ) and highly significant improvement $(\mathrm{P}<0.01)$ in self-reported functional disabilities measured by PSFS (PValue was 0.009) ROM at post treatment compared to pretreatment.

In United States, the popularly used laser based treatments are the infrared gallium-aluminium-arsenide and the visible helium-neon lasers. Due the ease of use, this combination is generally preferred. Moreover, advantage of the broader experimental background along with the cost effective availability may be another important reasons to use such combination [6]. Even though there is evident effect of laser irradiation on the metabolism and surface charges on cell culture, yet the ultra-structure is not disturbed through the use of such technology [7]. The number of studies pertaining to the aspect of laser irradiation and biological structure and function is increasing with time. Along with so many other recent issues, the impacts of the LLL analgesic in relation to musculoskeletal disorders are still an on-going argument.

In case of rheumatoid arthritis, myofascial pain syndrome, posttraumatic joint disorders and fibromyalgia, some reports are available pertaining to the superiority of the efficacy of LLL therapy in comparison to placebo [7]. However, beneficial effects in relation to the pain relief was not observed in all the cases, for instance, Krasheninnikoff et al. reported that in case of lateral epicondylitis LLL therapy was inferior compare to the provided placebo. Depending on the various painful musculoskeletal conditions LLL therapy showed variable efficacy which raised the controversy [8]. Probably due to various mechanisms such as positive effect on chondrocyte proliferation and matrix synthesis LLL has turned out to be an effective measure for LBP pain management [7]. Implementation and effectiveness of low power Ga-As pulse laser has been reported in relation to the function of the fibroblast cells and improvement of connective tissue damage. Relation was observed with the biostimulated effect of LLL at the cellular level during this tissue repairing process. Impact of LLL has been found in cytoplasmic enzyme activation, ATP production, consumption of oxygen and nucleic acids and protein synthesis [9].

Improvement in pain by VAS and functional disabilities by PSFS have proved the analgesic impact of laser and supported the report by Ozdemir et al. where reduction of spasm in muscular arterioles has been suggested that is essential for oxygenation of the tissues. Moreover, reports suggest that this enhances the ATP formation along with the regulation of the tissue metabolic rate considering reduced energy level. The other probable effect might be on the levels of the endorphin and pain associated gate controlling. All these possible mechanisms aid in interruption of the vicious cycle of the pain through laser treatment [6].

Another study suggested that the neuro-pharmacological analgesic effects of laser are may due to the release of serotonin and acetylcholine at the site [10].

This also is aligned with the findings of the study. There was a study to compare between three different treatments for mechanical low 
Page 5 of 5

back pain patients. He had total number of 150 patients of both sexes ranging from 21 to 64 years divided into three groups. The first group was treated by intensive dynamic back exercise program, the second group was treated by standardized traditional and physiotherapy program including isometric exercises for the back, the abdominal and leg muscles and training of muscle coordination; and the third group treated by a placebo control treatment that involves semi hot backs and light traction. The finding of the study showed that extension exercises were more effective in relieving pain than the other two groups. Females had better response to the intensive dynamic back exercise program than the placebo control program. Male patients treated with traditional physical therapy program included isometric exercise for trunk muscle responded significantly better than the placebo control program and the intensive dynamic back exercise program. The authors concluded that back extension exercises are the best in treating low back pain patients.

Machado et al. studied the randomized controlled trials related meta-analysis to understand the success rate of the McKenzie method with relation to the low back pain. They also conducted eleven trials for the same. McKenzie method abridged pain (weighted mean difference [WMD] on a $0^{\wedge}$ to 100 -point scale and scored -4.16 points following a $95 \%$ confidence interval, -7.12 to -1.20 ) and disability (WMD on a 0 to 100 -point scale, -5.22 points; $95 \%$ confidence interval, -8.28 to -2.16) at I week follow-up when compared with passive therapy for chronic LBP. When McKenzie method was comparatively estimated with advice to stay active, a decrease in disability favored advice (WMD on a 0 - to 100 -point scale, 3.85 points; $95 \%$ confidence interval, 0.30 to 7.39 ) at 12 weeks of follow-up was found [11].

The findings of this study agree with the conclusion of Ali et al. They reported the effectiveness of LPL therapy in 2003 with the purpose of pain reduction and managing functional disability for chronic LBP. In contrast to our results, reports are available mentioning no significant differences between laser and placebo laser treatments for this issue with relation to patients having acute and chronic low back pain due to lumbar disc herniation [12].

Similarly, Yousefi-Nooraie et al. performed a Cochrane review considering 7 studies with LLL for nonspecific low-back pain and not able to reach a firm conclusion due to the insufficiency of the data. Moreover, it was found that there is a requirement of more randomized controlled studies for the evaluation of the impact of the associated factors such as lengths of treatment, wavelengths and dosage $[13,14]$.

\section{Conclusion}

As mentioned in the result section, we did achieve significant differences in VAS and PSFS scales and ROM measure. LLL high significant in decrease pain and improve F.Pt standing, F-Pt walking and F-Asc-Desc. McKenzie technique high significant in improve ROM-F and F-Pt standing, F-Pt walking and F-Asc-Desc.

\section{References}

1. Ali G, Mehet K, Remzi C, Kemal N, Aysegul J, et al. (2003) Efficacy of low power laser therapy and exercise on pain and function in chronic low back pain. Laser in Surgery and Medicine 32: 233-238.

2. Andersson GBJ (1997) The epidemiology of spinal disorders. In: Frymoyer JW (ed). The adult spine: Principles and practice. Raven Press, New York. pp: 93-141.

3. Brown AW, Weber DC (2000) Physical agent modalitise In: Braddom RL (ed). Physical medicin and Rehabilitation. Harcourth Health Sciences Company, WB Saunders, London. pp: 440-458.

4. Fitz R (2001) Lasers and their therapeutic applications in chiropractic. Journal Canadian Chiropractic Association 45: 26-34.

5. Helen AC, Roger A, Christopher GM (2004) A systematic review of efficacy of McKenzie therapy for spinal pain. PM 50: 209-216.

6. Karu TI (2003) Biomedical photonic handbook. CRC press LLC, New York. pp: 1- 2.

7. Krasheninnikoff M, Ellitsgaard N, Rogvi-Hansen B, Zeuthen A, Harden $\mathrm{K}$, et al. (2000) No effect of low power laser in lateral epincondylitis. Scand J Rheumatol 23: 260-263.

8. Machado LA, de Souza MS, Ferreira PH, Ferreira ML (2006) The McKenzie method for low back pain: A systematic review of the literature with a meta-analysis approach. Spine 31: E254-262.

9. Mckenzie RA, May S (2003) The mechanical diagnosis and therapy of lumber disorders. Spina Publications Limited, Australia.

10. Ozdemir F, Birtane M, Koldno S (2001) The clinical efficacy of low power laser therapy upon pain and function in cervical osteoarthritis. Clinical Rheumatology 20: 181-184.

11. Schindl A, Schindl M, Pernerstorfer-Schon H, Schindl L (2000) Lowintensity laser therapy: A review. J Invest Med 48: 312-26.

12. Waddell G (1987) Volv award in clinical sciences. A new clinic model for the treatment of low back pain. Spine 12: 632-44.

13. Wilson-MacDonald J, Burt G, Griffin D, Glynn C (2005) Epidural steroid injection therapy for nerve root compression: A randomized, controlled trial. J Bone Joint Surg Br 87: 352-355.

14. Yousefi NR, Schonstein E, Heidari K, Rashidian A, Pennick V, et al. (2008) Low level laser therapy for nonspecific low-back pain. Cochrane Database Syst Rev. 